BULL. AUSTRAL. MATH. SOC.

VOL. 32 (1985), 379-387.

\title{
ON A CONJECTURE OF LITTLEWOOD IN DIOPHANTINE APPROXIIAATIONS
}

\section{S. KRASS}

\begin{abstract}
A conjecture of Littlewood states that for arbitrary $\underline{x}=\left(x_{1}, \ldots, x_{n}\right) \in \mathbb{R}^{n}, n \geq 2$, and any $\varepsilon>0$ there exist $m_{0} \neq 0, m_{1}, \ldots, m_{n}$ so that $\left|m_{0} \prod_{i=1}^{n}\left(m_{0} x_{i}-m_{i}\right)\right|<\varepsilon$. In this paper we show this conjecture holds for all $\underline{\xi}=\left(\xi_{1}, \ldots, \xi_{n}\right)$ such that $1, \xi_{1}, \ldots, \xi_{n}$ is a rational basis of a real algebraic number field of degree $n+1$.
\end{abstract}

\section{In troduction}

In a paper by Cassels and Swinnerton-Dyer in 1955, [2] they show that if $1, \alpha_{1}, \alpha_{2}$ is a basis (over $($ ) of a real cubic number field, then, for any $\varepsilon>0$, there exist integers $m_{0} \neq 0, m_{1}, m_{2}$ such that

$$
\left|m_{0}\left(m_{0} \alpha_{1}-m_{1}\right)\left(m_{0} \alpha_{2}-m_{2}\right)\right|<\varepsilon
$$

This result reinforces (but of course does not prove) for $n=2$ a conjecture by Littlewood that for arbitrary $\underline{x}=\left(x_{1}, \ldots, x_{n}\right) \in \mathbb{R}^{n}$, $n \geq 2$, and any $\varepsilon>0$ there exists $\underline{m}=\left(m_{0}, m_{1}, \ldots, m_{n}\right) \in \mathbb{Z}^{n+1}$ with $m_{0} \neq 0$ such that

Received 26 March 1985 ,

Copyright Clearance Centre, Inc. Serial-fee code: 0004-9727/85 $\$ A 2.00+0.00$. 


$$
\left|m_{0} \prod_{i=1}^{n}\left(m_{0} x_{i}-m_{i}\right)\right|<\varepsilon .
$$

In this paper we extend the Cassels, Swinnerton-Dyer result from $n=2$ to all $n \geq 2$. That is to say if $\underline{\xi}=\left(\xi_{1}, \ldots, \xi_{n}\right)$ with $1, \xi_{1}, \ldots, \xi_{n}$ a basis of $F$, a real number field of degree $n+1$, then, for any $\varepsilon>0$, there exists $\underline{m} \in \mathbb{Z}^{n+1}, m_{0} \neq 0$ such that

$$
\left|m_{0} \prod_{i=1}^{n}\left(m_{0} \xi_{i}-m_{i}\right)\right|<\varepsilon
$$

If $M$ is a full $\mathbb{Z}$-module in $F, R(M)$ denotes the coefficient ring of $M$. Namely

$$
R(M)=\{\alpha \in F: \alpha M \subseteq M\} .
$$

We will first need to prove the following lemma concerning units. in $R(M)$ which may be of independent interest.

LEMMA. $M$ is a fulz $\mathbb{Z}$-module in $F$ a real (algebraic) number field of degree $n+1$ and $R(M)$ the coefficient ring of $M_{0}$ For alz $\alpha=\alpha_{[0]} \in F, \alpha_{[j]}, j=0, \ldots, n$ denote the conjugates of $\alpha$ ordered so that $\alpha_{[j]} \in \mathbb{R}, j=0, \ldots, n-2 s, \quad \alpha_{[j]}=\bar{\alpha}_{[s+j]} \in \mathbb{C}, J=n-2 s+1, \ldots, n-s$ where $s$ is the number of pairs of complex conjugates. Then there exists an infinite sequence $\Gamma=\left(\gamma_{k} \in R(M), k=1,2, \ldots\right)$ with each $\gamma_{k} a$ zonit in $R(M)$ such that

(i) $\lim _{k \rightarrow \infty} \gamma_{k[j]} / \gamma_{k[n-s]}=1, j=1, \ldots, n-s-1$ (with $\left.\gamma_{k[j]}=\left(\gamma_{k}\right)_{[j]}\right)$ (ii) $\lim _{k \rightarrow \infty} \gamma_{k}=\infty$.

\section{Proof of Lemma}

By a theorem of Dirichlet the (multiplicative) group of units in $R(M)$ is generated by $n-s$ independent units, $[1, p, 112]$. Let $B_{i}, i=1, \ldots, n-s$ be $n-s$ independent units in $R(M)$. For $\varepsilon>0$ it is clear that the system of inequalities 
(2.1)

$$
\left|\sum_{i=1}^{n-s} x_{i} \log \right| \beta_{i[j]} / \beta_{i[n-s]}||<\varepsilon, j=1, \ldots, n-s-1 .
$$

has infinitely many (integer) solutions $\underline{x}=\underline{v}=\left(v_{1}, \ldots, v_{n-s}\right) \in \mathbb{Z}^{n-s}$.

Let $\varepsilon_{k}>0, k=1,2, \ldots$ with $\lim _{k \rightarrow 0} \varepsilon_{k}=0$ and let

$v_{k}=\left(v_{k 1}, \ldots, v_{k, n-s}\right) \in \mathbb{Z}^{n-s}$ be an integer solution of (2.1) for

$\varepsilon=\varepsilon_{k}, k=1,2, \ldots$ Then writing

$$
\psi_{k}=\prod_{i=1}^{n-s} B_{i}^{v_{k i}}, k=1,2,3, \ldots
$$

we have by construction

$$
\lim _{k \rightarrow \infty}\left|\psi_{k[j]} / \psi_{k[n-s]}\right|=1, j=1, \ldots, n-s-1
$$

Now we will write for all $k$ (with $e(x)=e^{\sqrt{-1} 2 \pi x}$ )

$$
\psi_{k[j]}=\left|\psi_{k[j]}\right| e\left(\theta_{k j}\right),-\frac{1}{2}<\theta_{k j} \leq \frac{1}{2}, j=0, \ldots, n .
$$

since $\psi_{k[j]} \in \mathbb{R}, j=0, \ldots, n-2 s$, replacing $\beta_{i}$ by $\beta_{i}^{2}, i=1, \ldots, n$, if necessary, we may assume without loss of generality.

$$
\theta_{k j}=0, j=0, \ldots, n-2 s .
$$

Now the infinite set of "amplitude" vectors

$$
\theta=\left\{\underline{\theta}_{k}=\left(\theta_{k, n-2 s+1}, \ldots, \theta_{k, n-s}\right): k=1,2, \ldots\right\}
$$

must contain at least one limit point $\phi=\left(\phi_{n-2 s+1}, \ldots, \phi_{n-s}\right)$, say, with $-\frac{1}{2} \leq \phi_{j} \leq \frac{1}{2}, j=n-2 s+1, \ldots, n-s$.

If $\phi=0$, then $r_{k}=\psi_{k}$ satisfies (i) of the lemma. So we suppose $\Phi \neq \underline{0}$. We may then choose an infinite subsequence of the $\psi_{k}$

$$
\rho_{p}=\psi_{k_{p}}, p=1,2, \ldots
$$

such that

$$
\lim _{p \rightarrow \infty} \rho_{p[j]}\left|\rho_{p[j]}\right|=e\left(\phi_{j}\right), j=n-2 s+1, \ldots, n-s .
$$

Finally we put 


$$
\gamma_{k}=\rho_{k+1} / \rho_{k}
$$

writing $\gamma_{k[j]}=\left|\gamma_{k[j]}\right| e\left(\phi_{k j}\right)$ it is clear that

$$
\lim _{k \rightarrow \infty} \phi_{k j}=0, j=n-2 s+1, \ldots, n-s .
$$

of course $\phi_{k j}=0, j=0, \ldots, n-2 s$ by (2.4).

Then we only need observe that, for $j=1, \ldots, n-s-1$,

$\left|\gamma_{k[j]} / \gamma_{k[n-s]}\right|=\left|\rho_{k+1,[j]} / \rho_{k+1,[n-s]}\right|\left|\rho_{k[j]} / \rho_{k[n-s]}\right| \rightarrow 1$, as $k \rightarrow \infty$ to see that $\gamma_{k}, k=1,2, \ldots$ satisfies $(i)$ of the lemma.

Now the (homogeneous) simultaneous equation system

$$
\left.\sum_{i=0}^{n-s} x_{i} \log \mid B_{i[j]}\right]_{i[n-s]} \mid=0, \quad j=1, \ldots, n-s-1
$$

has at least one of the $(n-s-1) \times(n-s-1)$ submatrices of $i$ ts coefficient matrix non-singular (since the regulator is non zero). So the solution set of (2.5) is the line, $L$, where

$$
L=\left\{\lambda \underline{y}: \in \mathbb{R} \text {, and } \underline{y} \neq \underline{0} \underline{y} \in \mathbb{R}^{n-s} \text { is a solution of }(2.5)\right\} \text {. }
$$

The set

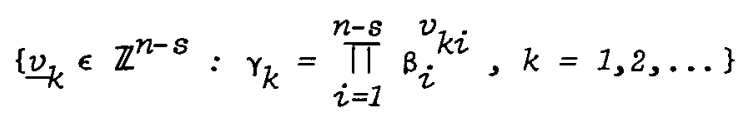

is a subset of solutions $\underline{x} \in \mathbb{Z}^{n-s}$ satisfying (2.1) and the elements are lattice points lying "near" the line $L$.

$$
\text { Suppose for the present } \prod_{i=1}^{n-s} B_{i} y_{i} \neq 1 \text {. Observe both } \underline{x}=\underline{y}
$$

and $\underline{x}=-\underline{y}$ satisfy (2.5). Hence we may choose $y$ with $\prod_{i=1}^{n-s} \beta_{i}{ }_{i}>1$.

Then for any $J>0$ there exists $\underline{v}_{k}$ near $\lambda \underline{y}$ for some $\lambda>J$, establishing (ii) of the lemma. So we need only show

$$
\prod_{i=1}^{n-s} B_{i}^{y_{i}} \neq 1 \text {, cony } \underline{y} \neq \underline{0}, \underline{y} \text { a solution to (2.5). }
$$

suppose $\prod_{i=1}^{n-s} \beta_{i} y_{i}=1$. Then it follows easily that there exist solutions 
$\underline{x}=\underline{v} \in \mathbb{Z}^{n-s}$ to (1) such that $|\theta|=\left|\prod_{i=1}^{n-s} \beta_{i}^{v}{ }^{v}\right| \approx 1$ and $\left|\theta_{[1]}\right| \approx \ldots \approx\left|\theta_{[n-s]}\right|$ where " $z$ " denotes equality up to any arbitrarily small fixed error. But $\left|\theta_{[n-2 s+j]}\right|=\left|\theta_{[n-s+j]}\right|, j=1, \ldots, s$ and $1=\prod_{j=0}^{n}\left|\theta_{[j]}\right|$, so $\prod_{i=1}^{n-s} B_{i}^{y_{i}}=1 \Rightarrow$ there exist irrational units $\theta$ with all conjugates arbitrarily near the unit circle. But this is impossible, see [3, p.137]. So (2.6) is established proving the lemma.

The following results follow trivially.

COROLLARY 1. The Zemma holds with (i) replaced by

$$
\lim _{k \rightarrow \infty} \gamma_{k[j]} / \gamma_{k[n]}=1, j=1, \ldots, n \text {. }
$$

COROLLARY 2. Both the Zerma and Corolzary 1 hold with

$$
\text { replaced by }
$$

$$
\lim _{k \rightarrow \infty} \gamma_{k}=0 \text {. }
$$

\section{Theorem}

Let $\xi=\left(\xi_{1}, \ldots, \xi_{n}\right)$ so that $1, \xi$ is a basis of $F$ a real number field of degree $n+1$. Then for any $\varepsilon>0$ there exist integers $m_{0} \neq 0, m_{1}, \ldots, m_{n}$ so that

$$
\left|m_{0} \prod_{j=1}^{n}\left(m_{0} \xi_{j}-m_{j}\right)\right|<\varepsilon
$$

Proof. Let $\xi_{0}=1$ and $\xi_{[i] j}=\left(\xi_{j}\right)_{[i]}, i, j=0, \ldots, n$ with conjugates ordered by the convention in the lemma. $A$ is the matrix

$$
A=\left(\xi_{[i] j}: i, j=0, \ldots, n\right) .
$$

It is well-known that $\operatorname{det} A \neq 0$. So we may define

$$
U=\left(u_{i j}: i, j=0, \ldots, n\right)=A^{-1} \text {. }
$$

By the row conjugate structure of $A$ we have 


$$
u_{i 0} \in F \text { and } u_{i j}=\left(u_{i 0}\right)_{[j]}, i, j=0, \ldots, n \text {. }
$$

Thus $u_{00}, \ldots, u_{n o}$ is a base of the full $\mathbb{Z}$-module

$$
M=\left\{\underline{m} \cdot \underline{u}_{0}=\sum_{i=0}^{n} m_{i} u_{i 0}: \underline{m}=\left(m_{0}, \ldots, m_{n}\right) \in \mathbb{Z}^{n+1}\right\} .
$$

We have used the notation

$$
\underline{u}_{j}=\left(u_{0 j}, \ldots, u_{n j}\right)^{t}=j-t h \text { column of } U, j=0, \ldots, n \text {. }
$$

By Corollary 1 there exists a sequence of units

$$
\Gamma=\left(\gamma_{k} \in R(M), k=1,2, \ldots\right) \text { such that }
$$

(3.1) $\lim _{k \rightarrow \infty} \gamma_{k[j]} / \gamma_{k[n]}=1, j=1, \ldots, n ;$ and $\lim _{k \rightarrow \infty} \gamma_{k}=\infty$.

For convenience, noting $u_{n o} \neq 0, u_{n o} \in M$, we write

$$
\mid \text { Norm } u_{n o}|=| \prod_{j=0}^{n} u_{n j} \mid=u>0 \text {. }
$$

Clearly $\gamma_{k} u_{n 0} \in M$, with $\mid$ Norm $\gamma_{k} u_{n o} \mid=u$, all $\gamma_{k} \in \Gamma$.

Let

$$
\mathbb{Z}(\Gamma)=\left\{\underline{m}=\underline{m}_{k} \in \mathbb{Z}^{n+1}: \underline{m}_{k} \cdot \underline{u}_{0}=\gamma_{k} u_{n 0}, \gamma_{k} \in \Gamma\right\}
$$

By (3.1) and this definition

(3. 2') $\left\{\begin{array}{l}\lim _{k \rightarrow \infty} \underline{m}_{k} \cdot \underline{u}_{j} / \underline{m}_{k} \cdot \underline{u}_{n}=u_{n j} / u_{n n}, j=1 \ldots, n \\ \lim _{k \rightarrow \infty}\left|\underline{m}_{k} \cdot \underline{u}_{0}\right|=\infty .\end{array}\right.$

Thus for any $\varepsilon>0$ and $K>1$ we have for all sufficiently large $k$

(3. 2') $\left\{\begin{array}{l}\underline{m}_{k} \cdot u_{j} / \underline{m}_{k} \cdot \underline{u}_{n}-u_{n j} / u_{n n}=\varepsilon_{k j},\left|\varepsilon_{k j}\right|<\varepsilon, j=1, \ldots, n \\ \left|\underline{m}_{k} \cdot \underline{u}_{0}\right|>k\end{array}\right.$

Since $v=\left|\prod_{j=0}^{n} \underline{m}_{k} \cdot \underline{u}_{j}\right|=\left|\underline{m}_{k} \cdot \underline{u}_{0}\right|\left|\underline{m}_{k} \cdot \underline{u}_{m}\right|^{n} \prod_{j=1}^{n-1}\left|\underline{m}_{k} \cdot \underline{u}_{j} / \underline{m}_{k} \cdot \underline{u}_{k}\right|$

it follows from $\left(3.2^{\prime}\right)$ for all $\underline{m}_{k} \in \mathbb{Z}(\Gamma)$ with $k$ sufficiently large

$$
u>K\left|\prod_{j=1}^{n-1} \frac{1}{2} u_{n j} / u_{n n}\right|\left|\underline{m}_{k} \cdot u_{n}\right|^{n} \text {. }
$$


So $\underline{m}_{k} \cdot \underline{u}_{n}=O\left(K^{-1 / n}\right)$ and then by $\left(3.2^{\circ}\right)$

(3. 3) $\underline{m}_{k} \cdot \underline{u}_{j}=O\left(K^{-1 / n}\right), j=1, \ldots, n, \underline{m}_{k} \in \mathbb{Z}(\Gamma)$ (sufficiently large) $k$. We note, and it is easily shown, that there are only finitely many $\underline{m} \in \mathbb{Z}(\Gamma)$ with $m_{0}=0$. So without loss of generality we suppose

$$
\underline{m} \in \mathbb{Z}(\Gamma) \Rightarrow m_{0}>0
$$

as (3.2) holds if we replace $\underline{m}_{k}$ by $-\underline{m}_{k}$.

Now suppose $\lim _{k \rightarrow \infty} \underline{m}_{k} \cdot \underline{u}_{0} / m_{k 0}=0$. Then writing

$$
\underline{w}_{k}=\left(w_{k 0}, \ldots, w_{k n}\right), \quad w_{k j}=m_{k} \underline{u}_{j} / m_{k 0}, j=0, \ldots, n
$$

we have by this assumption together with (3.3) and (3.4)

$$
\underline{0} \neq \lim _{k \rightarrow \infty} \underline{m}_{k} / m_{k 0}=\lim _{k \rightarrow \infty} \underline{w}_{k} U^{-1}=0 \text {. }
$$

By this contradiction we have shown there exists $w>0$ so that (3.5) $\left|\underline{m}_{k} \cdot \underline{u}_{0} / m_{k 0}\right|>w, \underline{m}_{k} \in \mathbb{Z}(\Gamma)$, alz (sufficiently Zarge) $k$.

$$
\begin{gathered}
u=\left|\underline{m}_{k} \cdot \underline{u}_{0} / m_{k 0}\right|\left|m_{k 0}^{1 / n} \underline{m}_{k} \cdot \underline{u}_{n}\right| \prod_{j=1}^{n-1}\left|\underline{m}_{k} \cdot \underline{u}_{j} / \underline{m}_{k} \cdot \underline{u}_{n}\right| . \\
\Rightarrow v>w\left|\prod_{j=1}^{n-1} \frac{1}{2} u_{n j} / u_{n n}\right|\left|m_{k 0}^{1 / n} \underline{m}_{k} \cdot \underline{u}_{n}\right|^{n} .
\end{gathered}
$$

So by the above result and the first part of (3.2) there exists $J>0$ so that

(3.6) $\left|m_{k 0}^{1 / n} \underline{m}_{k} \cdot \underline{u}_{j}\right|<J, j=1, \ldots, n, \underline{m}_{k} \in \mathbb{Z}(\Gamma)$, (sufficiently large) $k$. We now define an $n \times n$ submatrix of $U=A^{-1}$ by

$$
U_{*}=\left(u_{i j}: i, j=1, \ldots, n\right) \text {. }
$$

We note (and it is easily shown) that

$$
\operatorname{det} U_{*}=\operatorname{det} U \neq 0 \text {. }
$$

We define, for all $\underline{m} \in \mathbb{Z}^{n+1}$,

$$
h(\underline{m})=\left|m_{0}\right|^{1 / n}\left(m_{0} \xi_{1}-m_{1}, \ldots, m_{0} \xi_{n}-m_{n}\right)
$$


and note the identity

$$
\underline{h}(\underline{m}) U_{*}=-\left|m_{0}\right|^{1 / n}\left(\underline{m} \cdot \underline{u}_{1}, \ldots, \underline{m} \cdot \underline{u}_{n}\right) .
$$

For $\underline{m}_{k} \in \mathbb{Z}(\Gamma)$ we write

$$
\rho_{k}=\left(\rho_{k 1}, \ldots, \rho_{k n}\right), \quad \rho_{k j}=\left|m_{k 0}\right|^{1 / n} \underline{m}_{k} \cdot \underline{u}_{j}, j=1, \ldots, n .
$$

Then by $(3.8)$

$$
\underline{h}\left(m_{k}\right) U_{*}=-\rho_{k}=-\rho_{k n}\left(\rho_{k 1} / \rho_{k n}, \ldots, \rho_{k, n-1} / \rho_{k n,} 1\right) .
$$

BY

(3.2') $\rho_{k j} / \rho_{k n}=u_{n j} / u_{n n}+\varepsilon_{k j},\left|\varepsilon_{k j}\right|<\varepsilon, j=1, \ldots, n\left(\varepsilon_{k n}=0\right)$.

So

$$
\underline{h}\left(m_{k}\right) U_{*}=-\left(\rho_{k n} / u_{n n}\right)\left(u_{n 1}, \ldots, u_{n n}\right)-\rho_{k n}\left(\varepsilon_{k 1}, \ldots, \varepsilon_{k n}\right)
$$

and

$$
\underline{h}\left(m_{k}\right)=-\left(\rho_{k n} / u_{n n}\right)(0, \ldots, 0,1)-\left(\delta_{k 1}, \ldots, \delta_{k n}\right)
$$

since $\left(u_{n 1}, \ldots, u_{n n}\right) U_{*}^{-1}=(0, \ldots, 0,1)$ and we write

$$
\left(\delta_{k 1}, \ldots, \delta_{k n}\right)=\rho_{k n}\left(\varepsilon_{k 1}, \ldots, \varepsilon_{k n}\right) U_{*}^{-1} .
$$

Finally writing $\underline{h}\left(\underline{m}_{k}\right)=\underline{h}_{k}=\left(h_{k 1}, \ldots, h_{k n}\right)$ we observe

$$
\left|\prod_{j=1}^{n} h_{k j}\right|=\left|\prod_{j=1}^{n-1} \delta_{k j}\right|\left|\rho_{k n} / u_{n n}+\delta_{k n}\right| \rightarrow 0 \text { as } k \rightarrow \infty
$$

since by (3.6) $\rho_{k n} / u_{n n}=O(1)$ and by (3.2') and (3.6)

$$
\delta_{k j} \rightarrow 0, j=1, \ldots, n \text { as } k \rightarrow \infty .
$$

This completes the proof of the theorem.

\section{References}

[1] Borevich, Z.I. and Shafarevich, I.R., Number Theory, (Academic Press, 1966).

[2] Cassels, J.W.S. and Swinnerton-Dyer, H.P.F., "On the product of three homogeneous linear forms and indefinite ternary quadratic forms", Philos. Trans. Roy. Soc. London, Ser. A, 248 (1955), 73-96. 
[3] Pollard, H., The theory of algebraic numbers, (The Mathematical Association of America, 1950).

School of Mathematics,

University of New South wales,

Kensington,

N.S.W. 2033. 